\title{
Exciton surface states in molecular crystals*
}

\author{
Joseph Hoshen and Raoul Kopelman \\ Department of Chemistry, The University of Michigan, Ann Arbor, Michigan 48104 \\ (Received 26 February 1974) \\ A new Hamiltonian partition method, used previously for cooperative excitations in molecular \\ crystals, has been adopted for the treatment of surface exciton states in molecular crystals. The \\ formation of surface excitons depends on the relative magnitude of the exciton transfer integrals, $J$ \\ terms, as compared to the environmental shift integrals, $D$ terms. This was established for a sample \\ calculation on a simple cubic molecular crystal. It was found that when the absolute value of the \\ nearest neighbor $D$ term exceeds the corresponding $J$ term two localized states emerge for each \\ value of a two dimensional wavevector. The two localized states are degenerate in the limit of an \\ infinitely thick crystal. The localization of the surface states increases with an increase in the \\ magnitude of the $D$ term
}

\section{INTRODUCTION}

The existence of surface states in pure crystals has been proposed by Tamm, ${ }^{1}$ and later by Shockley. ${ }^{2}$ Following these pioneering works, surface states have been a subject of many investigations. Particularly worth noting are the comprehensive works of Koutecky ${ }^{3}$ and Grimley. ${ }^{4}$ These studies were mainly confined to electron states in intrinsic semiconductors. However, surface states in molecular crystals have drawn only limited attention. Recently, Stern and Green ${ }^{5}$ have explored the possibilities of surface states in naphthalene and anthracene. They have utilized an extended Grimley ${ }^{4}$ method in their investigation of surface electron states in naphthalene and anthracene crystal systems. Stern and Green's ${ }^{5}$ calculations are relatively involved and tend to be more complicated as more intermolecular interactions are taken into account.

In this paper we shall concentrate on some aspects of surface electronic excitons, rather than on the problem of surface electron states. Just as for some of the electron states, Frenkel ${ }^{6}$ exciton states are determined in the tight binding approximation. ${ }^{7}$ However, the major differences between the electron states and the Frenkel excitons is that when dealing with excitons multiple electron wavefunctions are employed. (In the electron theory in the tight binding approximation only a single electron crystal orbital is constructed.) The introduction of multiple electron wavefunctions in the exciton case gives rise to intermolecular interaction terms which do not have a direct counterpart in electron state theory. These terms are the environmental shift term (the $D$ term) and the exciton transfer term ( $J$ term). In the light of the preceding discussion the following questions are pertinent:

(a) The localization problem: The Frenkel-Davydov $^{6,7}$ theory predicts the formation of bulk states in molecular crystals corresponding to the various values of the wavevector $(\mathrm{K})$. An interesting question in this context is whether the simple single band Davydov theory can be extended to account for localized surface states.

(b) Density of surface states: It is interesting to inquire whether surface states form a continuous exciton band in two dimensions. The question is whether local- ized states are formed for all values of the two dimensional $\mathbf{k}$ vector, or are there gaps in the energy dispersion curves which might lead to forbidden zones in the density-of-states in two dimensions.

(c) Energy trapping: Just as in the case of impurity trapping, localized surface states could trap bulk excitons. Since surface localized states form extended states in two dimensions, it is worthwhile to investigate the possibility of energy surface transport.

(d) Optical properties: Optical absorption is generally associated with the bulk of the crystal, however, the correlation of absorption with crystal thickness is of interest. On the other hand, reflection is more closely related to surfaces, so that surface states may effect the reflecting properties of the crystal.

In the following sections we shall utilize a new method for treating surface states. Although we confine our interests to molecular excitons, the method can be projected to other elementary excitations, like phonons where the tight binding approximation is applicable. In Sec. II we shall apply the Hamiltonian partition method to surface perturbation. This method was used by Hoshen and Jortner ${ }^{\mathcal{\theta}}$ in determining the properties of cooperative excitons. Section III is devoted to the solution of a simple problem utilizing the result of Sec. II. In Sec. IV, some numerial results are displayed for the simple model of Sec. III.

\section{THEORY}

Consider a molecular crystal containing $N$ unit cells with one molecule per unit cell. There are $N_{1}, N_{2}$, and $N_{3}$ unit cells in the direction of each of the primitive vectors: $\mathrm{a}_{1}, \mathrm{a}_{2}, \mathrm{a}_{3}$, respectively, so that $N=N_{1} N_{2} N_{3}$. We shall first assume $N_{1} \rightarrow \infty$ and $N_{2} \rightarrow \infty$, while $N_{3}$ is finite. Thus we may invoke cyclic boundary conditions for the plane spanned by $a_{1}$ and $a_{2}$. We can define symmetry wavefunctions for the lattice plane parallel to $a_{1}$ and $\mathrm{a}_{2}$ primitive vectors:

$$
|\mathbf{k}, l\rangle=\left(N_{1} N_{2}\right)^{-1 / 2} \sum_{n} e^{i \mathbf{k} \cdot \mathbf{R}_{n}}\left|\mathbf{R}_{n}, l\right\rangle,
$$

where $\left|\mathrm{R}_{n}, l\right\rangle$ is a localized excitation in the $l$ th plane. $R_{n}$ denotes the coordinate of a molecule in the $l$ th plane and is given by 


$$
\begin{aligned}
\mathrm{R}_{n} & =n_{1} \mathrm{a}_{1}+n_{2} \mathrm{a}_{2}, & & \\
n_{1} & =0,1, \ldots, N_{1}-1 & & \left(N_{1}-\infty\right) \\
n_{2} & =0,1, \ldots, N_{2}-1 & & \left(N_{2}-\infty\right) . \\
l & =0,1, \ldots, N_{3}-1, & &
\end{aligned}
$$

$$
\left|\mathrm{R}_{n}, l\right\rangle=a \varphi^{f}\left(\mathrm{R}_{n}, l\right) \Pi^{\prime} \varphi^{0}\left(\mathbf{R}_{n}^{\prime}, l\right) \quad \mathbf{R}_{n}^{\prime}, l^{\prime} \neq \mathrm{R}_{n}, l,
$$

where $a$ is the antisymmetrization operator permuting electrons among molecules, and $f$ denotes an excited state of the molecule. The wavevector $\mathbf{k}$ is defined in two dimensions only.

It is convenient to define a crystal Hamiltonian $\hat{H}$ relative to the ground state.

$$
\hat{H}=\hat{\mathscr{K}}-E_{0} \text {, }
$$

where $\hat{\mathcal{H}}$ is the cryslal Hamiltonian and $E_{0}$ is the ground state energy in the first order. Since $\hat{H}$ is diagonal in $\mathbf{k}$ we can restrict this discussion to operators in the $\mathbf{k}$ space only.

At this point we shall write down the matrix elements of $\hat{H}(\mathbf{k})$. The diagonal element of $\hat{H}(\mathbf{k})$ is given by

$[\mathbf{H}(\mathbf{k})]_{l l} \equiv\langle l|\hat{H}(\mathbf{k})| l\rangle=\epsilon^{f}+\sum_{l^{\prime}=0}^{N_{3}-1} \bar{D}_{l l^{\prime}}+\sum_{\mathbf{R}_{n}} J\left(\mathbf{R}_{n}\right) e^{i \mathbf{k R}_{n}}$,

where $\epsilon^{f}$ is the gas phase excitation energy of a single molecule and $\mathrm{R}_{n}=n_{1} \mathrm{a}_{1}+n_{2} \mathrm{a}_{2}$, implying that the summation over the $J$ terms is carried in the $l$ th plane. The exciton transfer term is

$$
J\left(\mathbf{R}_{m}\right)=\left\langle\boldsymbol{\alpha} \varphi^{f}(\mathbf{O}) \varphi^{0}\left(\mathbf{R}_{m}\right)\left|W\left(\mathbf{O}, \mathbf{R}_{m}\right)\right| \varphi^{0}(\mathbf{O}) \varphi^{f}\left(\mathbf{R}_{m}\right)\right\rangle,
$$

where $W\left(\mathbf{O}, \mathbf{R}_{m}\right)$ denotes electron interactions between a molecule at the origin and a molecule at site $\mathbf{R}_{m}$. The $\vec{D}_{l l}$, terms in Eq. (5) are planewise summations of the environmental shift terms and given by

$$
\bar{D}_{l l^{\prime}}=\sum_{\mathrm{R}_{n}} D\left(\mathbf{R}_{l-l^{\prime}}+\mathbf{R}_{n}\right)
$$

again the summation is carried over $R_{n}=n_{1} a_{1}+n_{2} a_{2}$, but the interactions are between molecules of different planes for $l \neq l^{\prime}$. The environmental shift terms $D\left(\mathbf{R}_{m}\right)$ are

$$
\begin{aligned}
& D\left(\mathbf{R}_{m}\right) \\
& \quad=\left\langle\propto\left[\varphi^{f}(\mathbf{O}) \varphi^{f}(\mathbf{O})-\varphi^{0}(\mathbf{O}) \varphi^{0}(\mathbf{O})\right]\left|W\left(\mathbf{O}, \mathbf{R}_{m}\right)\right| \alpha \varphi^{0}\left(\mathbf{R}_{m}\right) \varphi^{0}\left(\mathbf{R}_{m}\right)\right\rangle .
\end{aligned}
$$

The off-diagonal elements of $\hat{H}(\mathbf{k})$ are given by

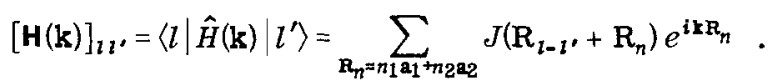

There is no simple solution for the eigenvalue problem of $\hat{H}(\mathbf{k})$ since no cyclic boundary condition was imposed in the $a_{3}$ direction. However, we shall define a perturbation matrix $\mathbf{V}(\mathbf{k})$, and an unperturbed matrix $\mathbf{H}_{0}(\mathbf{k})$ so that

$$
\mathbf{V}(\mathbf{k})=\mathbf{H}(\mathbf{k})-\mathbf{H}_{0}(\mathbf{k})
$$

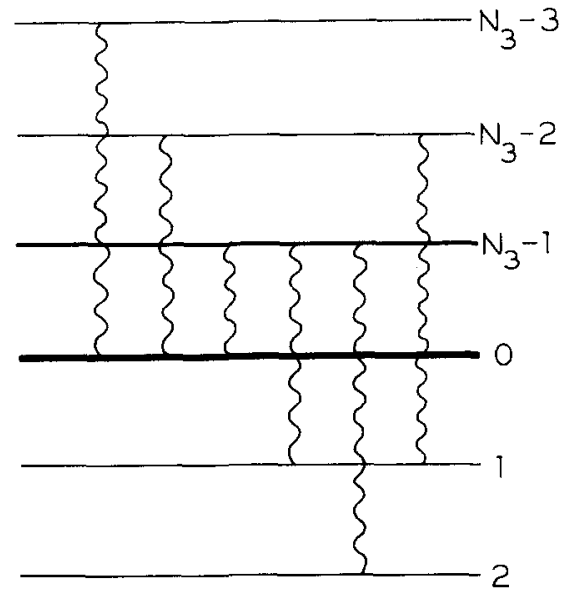

FIG. 1. Surface perturbation scheme: Crystal planes are designated by solid lines, surface planes are emphasized as heavy solid lines. The missing interactions between top and bottom crystal planes, required to establish the cyclic boundary condition for the $a_{3}$ direction, are denoted by wavy lines.

$\mathbf{H}_{0}(\mathbf{k})$ will be constructed in a form for which the cyclic boundary condition is satisfied in the $a_{3}$ direction, and thus can be easily diagonalized. The $\mathbf{H}_{\mathbf{0}}(\mathbf{k})$ will have this form if the $\left(N_{3}-1\right)$ th plane is adjacent to the first plane $\left(l_{3}=0\right)$. In this case all the diagonal elements of $\mathbf{H}_{0}(\mathbf{k})$ will be equal, while off-diagonal elements of $\mathbf{H}_{0}(\mathbf{k})$ are translationally equivalent in the $a_{3}$ direction. At this point we shall make an assumption which is necessary to simplify calculations. We shall assume that the planewise summation in Eq. (9) over the $J$ terms and over the $D$ terms in Eq. (7) is small and can be set to zero for sufficiently large but finite separations between $l$ and $l^{\prime}$ planes. We really need not worry about the $D$ terms because due to their nature they correspond to short range interactions (comparable to van der Waals type interactions). Hence we may take into account interactions between nearest neighbors only. However, the $J$ terms may correspond to long range transitiondipole-transition-dipole interactions for strong optical transitions. It is well known that dipole-dipole interactions do not converge for all values of $\mathbf{k}$, when a summation is carried over a sphere. ${ }^{9}$ However, it has been shown by Wette and Schacher, ${ }^{10}$ and recently by Philpott $^{11}$ that the planewise summation of the dipole-dipole interactions converges rapidly with the increase of distance between a molecule and a plane containing the molecules interacting with that molecule. To sum up, our assumption is generally justified, and we can assume zero interactions between a molecule and a plane that is $r$ crystal planes away from that molecule. The value of $r$ depends on the crystal and the crystal plane in question. 11

We shall illustrate the formation of a $\mathbf{V}(\mathbf{k})$ matrix by inspecting the diagram in Fig. 1. The wavy lines in Fig. 1 denote the interactions that are to be added to $\mathbf{H}(\mathbf{k})$ in order that cyclic boundary conditions are to be fulfilled for $H_{0}(k)$. In addition to the off-diagonal interactions, there are contributions to the diagonal elements of $\mathbf{H}_{0}(\mathbf{k})$ arising from the difference between the bulk $D$ terms and the corresponding surface $D$ terms. Under those condi- 
tions the $\mathbf{V}(\mathrm{k})$ matrix will have the following form:

$$
\mathbf{V}(\mathbf{k})=\left(\begin{array}{ccc}
\Delta \downarrow & w(k) & 0 \\
w(k)^{\dagger} & \Delta \uparrow & 0 \\
0 & 0 & 0
\end{array}\right)
$$

$\mathbf{w}(\mathbf{k})$ is a matrix of dimension $r$ whose elements are given by

$$
\mathbf{W}(\mathbf{k})_{\left(N_{3}-i\right), j}=-\sum_{\mathbf{R}_{n}} J\left[(j+i) \mathbf{a}_{2}+\mathbf{R}_{n}\right] e^{i \mathbf{k} \mathbf{R}_{n}}
$$

where $1 \leqslant i \leqslant r, 0 \leqslant j \leqslant r-1$, and $i+j \leqslant r$. The matrix $\mathbf{W}(\mathbf{k})$ is a triangular matrix. The diagonal elements of the diagonal matrices $\Delta \uparrow$ and $\Delta \downarrow$ are given by

$$
\begin{aligned}
& \Delta \downarrow_{j, j}=-\sum_{i=1}^{r-j} D_{j,\left(N_{3}-l\right)} \\
& \Delta \downarrow_{\left(N_{3}-j\right),\left(N_{3}-j\right)}=-\sum_{l=0}^{r-j} D_{\left(N_{3}-j\right), l} .
\end{aligned}
$$

The indices of $\boldsymbol{w}(\mathbf{k})$ and $\Delta$ matrices denote crystal planes rather than a position in the matrix array.

Once the form of Eq. (11) is established, the KosterSlater ${ }^{12}$ Green's function formalism can be utilized. The eigenvalues of the problem can be determined from the $2 r \times 2 r$ determinantal equation.

$$
D(E)=\left|\mathbf{1}_{a}-\mathbf{G}_{a a}(E) \mathbf{v}_{a a}(\mathbf{k})\right|,
$$

where $\mathbf{V}_{a a}(\mathbf{k})$ is a $2 r \times 2 r$ submatrix of $\mathbf{V}(\mathbf{k})$ represented in the form:

$$
\mathbf{V}_{a a}(\mathbf{k})=\left(\begin{array}{cc}
\Delta \dagger & \mathbf{w}(\mathbf{k}) \\
\mathbf{W}(\mathbf{k})^{\dagger} & \Delta \uparrow
\end{array}\right)
$$

and $\mathbf{G}_{a a}(E)$ is the corresponding Green's function submatrix. Utilizing the Dyson identity:

$$
\hat{G}=\hat{G}_{0}+\hat{G}_{0} \hat{V} \hat{G}
$$

the following equation can be derived for the perturbed Green's function ${ }^{13}$ :

$$
\hat{G}=\hat{G}_{0}+\hat{G}_{0} \hat{V}\left(\hat{1}-\hat{G}_{0} \hat{V}\right)^{-1} \hat{G}_{0} .
$$

This expression is especially useful for the extended states of the perturbed system.

\section{APPLICATION TO THE (001) PLANE OF A SIMPLE CUBIC CRYSTAL WITH NEAREST NEIGHBOR INTERACTIONS}

The Hamiltonian matrix elements Eqs. (5) to (7) for a simple cubic crystal with an inversion center containing one molecule per unit cell and nearest neighbor interactions only, for $l(\neq 0) \neq N_{3}-1$, are given by

$$
H(\mathbf{k})_{l l} \equiv\langle l|\hat{H}(\mathbf{k})| l\rangle=\epsilon^{f}+6 D+2 J\left(\cos \mathbf{k} \cdot a_{1}+\cos \mathbf{k} \cdot a_{2}\right),
$$

where $D$ and $J$ are the environmental shift, and the exciton transfer interaction between two adjacent molecules, respectively. The diagonal elements of $\mathbf{H}(\mathbf{k})$ for $l=0=N_{3}-1$ are

$$
H(\mathrm{k})_{l l}=\epsilon^{f}+5 D+2 J\left(\cos \mathrm{k} \cdot \mathrm{a}_{1}+\cos \mathrm{k} \cdot \mathrm{a}_{2}\right) .
$$

The off-diagonal elements are

$$
H(\mathbf{k})_{l, l \pm 1}=J \text {. }
$$

All other off-diagonal elements are zero. The perturbation matrix $\mathbf{v}_{a a}(\mathbf{k})$ [see $\mathrm{Eq}$. (16)] is given in the form:

$$
\mathbf{v}_{a a}(\mathbf{k})=\left(\begin{array}{cc}
-D & -J \\
-J & -D
\end{array}\right) \text {. }
$$

The secular determinant Eq. (15) can be recast in the form:

$D(E)=\left|\begin{array}{cc}1+g_{0}(E) D+g_{1}(E) J & g_{0}(E) J+g_{1}(E) D \\ g_{0}(E) J+g_{1}(E) D & 1+g_{0}(E) D+g_{1}(E) J\end{array}\right|$.

$g_{0}(E)$ and $g_{1}(E)$ are the diagonal, and off-diagonal (corresponding to adjacent planes) matrix elements of the unperturbed Green's function $G_{0}(E)$, where the energy $E$ is given relative to

$$
\epsilon^{f}+6 D+2 J\left(\cos \mathbf{k} \cdot \mathrm{a}_{1}+\cos \mathbf{k} \cdot \mathrm{a}_{2}\right)
$$

and $g_{0}(E)$ and $g_{1}(E)$ are given by

$$
\begin{aligned}
& g_{0}(E) \equiv N_{3}^{-1} \sum_{k_{3}}\left[E-2 J \cos \left(k_{3} a_{3}\right)\right]^{-1} \\
& g_{1}(E) \equiv N_{3}^{-1} \sum_{k_{3}} e^{i k_{3} a_{3}} /\left[E-2 J \cos \left(k_{3} a_{3}\right)\right],
\end{aligned}
$$

where $k_{3}=2 \pi P / N_{3} ; P=0,1, \ldots,\left(N_{3}-1\right)$.

Without loss of generality we shall set $J=\frac{1}{2}$ in order to simplify formulas. In the limit $N_{3} \rightarrow \infty, g_{0}(E)$, and $g_{1}(E)$ can be further simplified to the following expressions (replacing summations by integrations over $k_{3}$ ): for $E<-1$

$$
\begin{aligned}
& g_{0}(E)=-1 / \sqrt{E^{2}-1} \\
& g_{1}(E)=-\left(E+\sqrt{E^{2}-1}\right) / \sqrt{E^{2}-1} ;
\end{aligned}
$$

for $E>1$

$$
\begin{aligned}
& g_{0}(E)=1 / \sqrt{E^{2}-1} \\
& g_{1}(E)=\left(E-\sqrt{E^{2}-1}\right) / \sqrt{E^{2}-1} ;
\end{aligned}
$$

and for $-1<E<1$ a complex solution is obtained:

$$
\begin{aligned}
& g_{0}(E)=i / \sqrt{1-E^{2}} \\
& g_{1}(E)=\left(i E / \sqrt{1-E^{2}}\right)-1
\end{aligned}
$$

The eigenvector $C^{s}(\mathbf{k})$ of the Hamiltonian for the localized surface states $\psi^{s}(\mathbf{k})$ can be determined from the nonhomogeneous equation:

$$
\left(\begin{array}{cc}
1+g_{0}(E) D+\frac{1}{2} g_{1}(E) & g_{1}(E) D+\frac{1}{2} g_{0}(E) \\
g_{1}(E) D+\frac{1}{2} g_{0}(E) & 1+g_{0}(E) D+\frac{1}{2} g_{1}(E)
\end{array}\right)\left(\begin{array}{c}
C_{N_{3}-1}^{S}(\mathbf{k}) \\
C_{0}^{S}(\mathbf{k})
\end{array}\right)=\left(\begin{array}{l}
0 \\
0
\end{array}\right) .
$$

The determinantal Eq. (23) can be reduced to two equations in $E$ :

$$
\begin{aligned}
& 1+\left[g_{0}(E)+g_{1}(E)\right]\left(D+\frac{1}{2}\right)=0, \\
& 1+\left[g_{0}(E)-g_{1}(E)\right]\left(D-\frac{1}{2}\right)=0 .
\end{aligned}
$$

Utilizing Eqs. (32) and (33) we obtain

$$
C_{0}^{s}(\mathbf{k})=C_{\left(N_{3}-1\right)}^{s}(\mathbf{k}) \text {. }
$$

While employing Eqs. (32) and (34) the following relationship is given for the coefficients: 


$$
C_{0}^{s}(\mathbf{k})=-C_{\left(N_{3}-1\right)}^{s}(\mathbf{k})
$$

The normalization of the coefficients for Eq. (35a) is given $^{8}$ by

$$
\left[C_{0}^{S}(\mathbf{k})\right]^{2}=\left\{2\left(D+\frac{1}{2}\right)^{2}\left[g_{0}^{\prime}(E)+g_{1}^{\prime}(E)\right]\right\}^{-1},
$$

where

$$
g_{0}^{\prime}(E)=d g_{0}(E) / d E
$$

and

$$
g_{1}^{\prime}(E)=d g_{1}(E) / d E .
$$

The normalization of the coefficients of Eq. (35b) is given by

$$
\left[C_{0}^{s}(\mathbf{k})\right]^{2}=\left\{2\left(D-\frac{1}{2}\right)^{2}\left[g_{0}^{\prime}(E)-g_{1}^{\prime}(E)\right]\right\}^{-1} .
$$

It is very easy to give a simple expression for the localized states for $N_{3} \rightarrow \infty$. Utilizing Eqs. (26) and (27) [or Eqs. (28) and (29)] and Eqs. (33) and (34) twofold degenerate solutions are obtained, one for each of the equations (33) and (34). The two degenerate localized states, formed for $|D|>\frac{1}{2}$ and for $-1>E>1$, are given by:

$$
E_{s}=-D-1 /(4 D) \text {. }
$$

The expansion coefficients $C_{0}$ for the surface planes are

$$
\left[C_{0}^{s}(\mathbf{k})\right]^{2}=\frac{1}{2}\left[1-1 /\left(4 D^{2}\right)\right] .
$$

The other coefficients of the surface states wavefunctions

$$
\psi^{s}(\mathbf{k})=\sum_{l=0}^{N_{3}-1} C_{l}^{s}(\mathbf{k})|\mathbf{k}, l\rangle
$$

can be determined from the matrix equation ${ }^{8}$ :

$$
\begin{aligned}
& \left(\begin{array}{cc}
1_{a}-\mathbf{G}_{a a}(E) \mathbf{V}_{a a}(\mathbf{k}) & \mathbf{0} \\
-\mathbf{G}_{b a}(E) \mathbf{V}_{a a}(\mathbf{k}) & \mathbf{1}_{b}
\end{array}\right)\left(\begin{array}{c}
\mathbf{C}_{a}^{s}(\mathbf{k}) \\
\mathbf{C}_{b}^{s}(\mathbf{k})
\end{array}\right)=(\mathbf{0}), \\
& \mathbf{C}_{a}^{s}(\mathbf{k})=\left(\begin{array}{c}
C_{\left(N_{3}-1\right)}^{s}(\mathbf{k}) \\
C_{0}^{s}(\mathbf{k})
\end{array}\right),
\end{aligned}
$$

and

$$
\mathbf{C}_{b}^{s}(\mathbf{k})=\left(\begin{array}{c}
C_{1}^{s}(\mathbf{k}) \\
C_{2}^{s}(\mathbf{k}) \\
\cdot \\
\cdot \\
C_{\left(N_{3}-2\right)}^{s}(\mathbf{k})
\end{array}\right)
$$

Since $\mathbf{V}_{a d}(\mathbf{k})$ is independent of $\mathbf{k}$ [see Eq. (22)], we shall omit $\mathbf{k}$ from subsequent calculations. The following relations are obtained for the expansion coefficients:

$$
\begin{aligned}
& C_{n}^{s}=-\left\{\left[g_{n+1}(E) D+\frac{1}{2} g_{n}(E)\right] C_{N_{3}-1}^{s}+\left[\frac{1}{2} g_{n+1}(E)+g_{n}(E) D\right] C_{0}^{s}\right\}, \\
& C_{N_{3}-1}^{s}=-\left\{\left[g_{0}(E) D+\frac{1}{2} g_{1}(E)\right] C_{N_{3}-1}^{s}+\left[\frac{1}{2} g_{0}(E)+g_{1}(E) D\right] C_{0}^{s}\right\}, \\
& C_{0}^{s}=-\left\{\left[g_{1}(E) D+\frac{1}{2} g_{0}(E)\right] C_{N_{3}-1}^{s}+\left[\frac{1}{2} g_{1}(E)+g_{0}(E) D\right] C_{0}^{s}\right\}
\end{aligned}
$$

These expressions yield a simple solution for $N_{3}-\infty$. For the symmetric wavefunction [solution of Eq. (33)] we get from Eq. (38) and the above equations:

$$
C_{l}^{S}=C_{N_{3}-l-1}^{s}=(-1 / 2 D)^{l} C_{0}^{s}
$$

and for the antisymmetric wave function [solution of Eq. (34)]

$$
C_{l}^{s}=-C_{N_{3}-l-1}^{s}=(-1 / 2 D)^{l} C_{0}^{s} .
$$

The transition dipole matrix element for the surface states is [from Eq. (40)]:

$$
\mu^{s}=\sum_{l=0}^{N_{3}-1} C_{l}^{s}\left\langle\psi_{0}|\hat{\mu}| \mathbf{k}=0, l\right\rangle,
$$

where $\psi_{0}$ is the ground state wavefunction. Inserting the coefficients derived by Eq. (44) in Eq. (47) we obtain for the symmetric wavefunction:

$$
\mu^{s}=2 \mu_{0}\left(D+\frac{1}{2}\right) C_{0}^{s} \sqrt{N_{1} N_{2}} /[E-E(O)],
$$

where $E(O)$ is the eigenvalue for the 'unperturbed' system for $K=O$ and $\mu_{0}$ is the transition dipole for a single molecule from the ground state to state $f$. The transition dipole for the antisymmetric wavefunction is zero. This is anticipated since our system has an inversion symmetry. At this point it is worth mentioning the fact that the twofold degeneracy is accidental, since the two surface wavefunctions belong to two different group representations, i.e., symmetric and antisymmetric representations.

The optical absorption per molecule is directly proportional to the dipole strength per unit energy per molecule $d(e)$, where $d(E)$ is given for the localized surface states by

$$
d(E)=N^{-1}\left|\left\langle\psi_{0}|\hat{\mu}| \psi^{s}(0)\right\rangle\right|^{2} \delta\left(E-E_{s}\right) .
$$

Substituting the transition dipole matrix element from Eq. (48) for the symmetric wavefunction a simple expression is obtained for $d(E)$, with the help of Eq. (36):

$$
d(E)=\frac{-2 \mu_{0}^{2} \delta\left(E-E_{s}\right)}{N_{3}[E-E(O)]^{2}\left[g_{0}^{\prime}\left(E_{s}\right)+g_{1}^{\prime}\left(E_{s}\right)\right]} .
$$

This expression yields a simple result for $N_{3} \rightarrow \infty$ :

$$
d(E)=\frac{2 \mu_{0}^{2}}{N_{3}} \frac{2 D-1}{2 D+1} \delta[E+D+1 /(4 D)] .
$$

All the complications encountered in the calculation of the expansion coefficients can be avoided, if the Green's function is calculated, rather than the wavefunctions. In addition extended states are handled in a natural way by the Green's function method, on the same footing as the discrete states. The dipole strength per unit of energy per molecule is given in the Green's function representation by ${ }^{13}$ :

$$
d(E)=(1 / \pi N) \operatorname{Im}\left\langle\psi_{0}|\hat{\mu} G(E) \hat{\mu}| \psi_{0}\right\rangle .
$$

Utilizing Eqs. (18) and (52) the following expression is derived for $d(E)$ for a finite $N_{3}$ (see Appendix):

$$
\begin{aligned}
d(E) & =-\frac{1}{\pi N_{3}} \operatorname{Im} \frac{2\left(D+\frac{1}{2}\right) \mu_{0}^{2}}{\left\{1+\left[g_{0}(E)+g_{1}(E)\right]\left(D+\frac{1}{2}\right)\right\}[E-E(O)]^{2}} \\
& =\frac{-2 \mu_{0}^{2}}{N_{3}} \frac{\delta\left(E-E_{s}\right)}{[E-E(O)]^{2}\left[g_{0}^{\prime}\left(E_{s}\right)+g_{1}^{\prime}\left(E_{s}\right)\right]} .
\end{aligned}
$$

This expression is identical to Eq. (50) as expected. When $N_{3} \rightarrow \infty$, the eigenvalues of $\mathbf{H}_{0}(\mathbf{k})$ do not form a dis- 


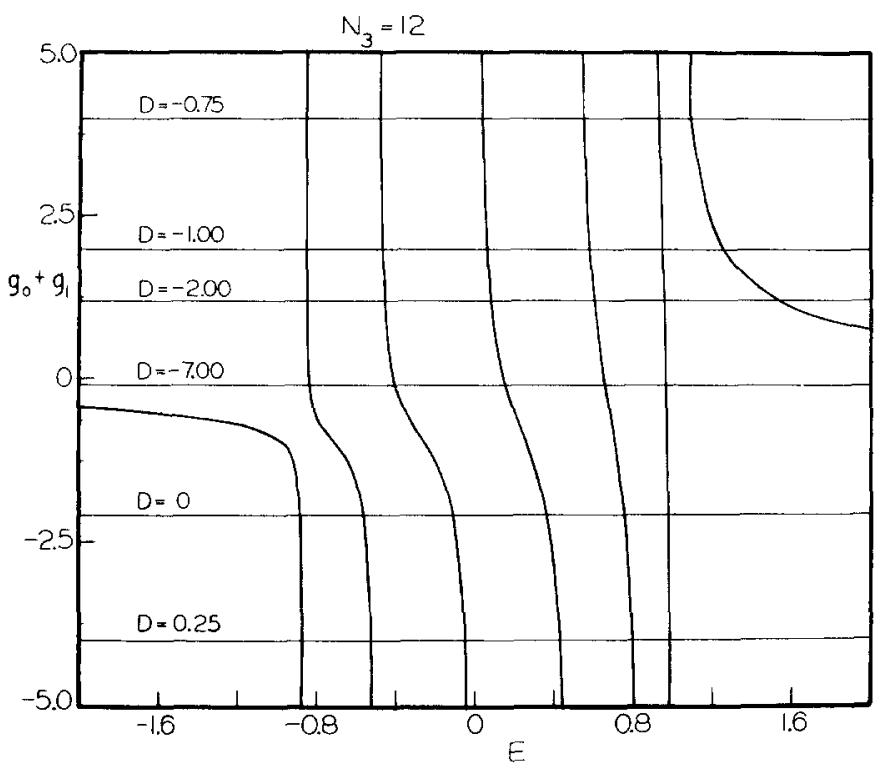

FIG. 2. A graphical solution of Eq. (33). The horizontal lines denote $y=-1 /(D+1 / 2)$.

crete set on the real axis. The discrete eigenvalues of $\hat{G}_{0}(E)$ form isolated poles on the real axis, but when $N_{3}-\infty, G_{0}(E)$ is characterized by a branch cut on the real axis for the eigenvalues of $\mathbf{H}_{0}(\mathbf{k})$. Hence the dipole strength per unit of energy per molecule can be recast in the form:

$$
\begin{aligned}
d(E)=-\lim _{N_{3} \rightarrow \infty} \frac{1}{\pi N_{3}} \frac{2(D}{\left[\begin{array}{l}
2 \\
2
\end{array}\right) \mu_{0}^{2}} \\
+E-E(\mathbf{O})]^{2}\left\{1+\left[g_{0}^{\prime}(E)+g_{1}^{\prime}(E)\right]\left(D+\frac{1}{2}\right)\right\} \\
+\mu_{0}^{2} \delta[E-E(\mathbf{O})] .
\end{aligned}
$$

The first term in Eq. (54) is due to surface perturbation and is zero in the limit $N_{3} \rightarrow \infty$. The second term in Eq. (54) denotes bulk absorption. Combining Eqs. (54), (30), and (31), we obtain for $-1<E<1$ :

$$
\begin{aligned}
d(E)=\frac{\mu_{0}^{2}}{\pi N_{3}} \operatorname{Im}[ & \frac{4\left(D^{2}-\frac{1}{4}\right)}{(1-E)\left(4 D E+4 D^{2}+1\right)} \\
& \left.+\frac{i 4\left(D+\frac{1}{2}\right)^{2} \sqrt{(1+E) /(1-E)}}{(1-E)\left(4 D E+4 D^{2}+1\right)}\right] \\
= & \frac{4 \mu_{0}^{2}\left(D+\frac{1}{2}\right)^{2} \sqrt{(1+E) /(1-E)}}{\pi N_{3}(1-E)\left(4 D E+4 D^{2}+1\right)} .
\end{aligned}
$$

As evident from Eq. (55), there are no virtual states ${ }^{13}$ in the region for which $-1<E<1$. The expression derived for $d(E)$ in the region $-1>E>1$ is given by

$$
d(E)=\frac{2 \mu_{0}^{2}(2 D-1)}{N_{3}(2 D+1)} \delta\left(E+D+\frac{1}{4 D}\right) .
$$

This expression is identical with Eq. (51), as expected.

The energy dispersion curves for the surface states are of the form:

$$
E^{s}(\mathbf{k})=E_{s}+\epsilon^{f}+6 D+\cos \left(\mathbf{k} \cdot \mathbf{a}_{1}\right)+\cos \left(\mathbf{k} \cdot \mathbf{a}_{2}\right) .
$$

Here the reference energy is again the crystal ground state. Utilizing Eq. (38) the following expression is obtained in the limit $N_{3} \rightarrow \infty$ :

$$
E^{s}(\mathbf{k})=\epsilon^{f}+5 D-1 /(4 D)+\cos \left(\mathbf{k} \cdot a_{1}\right)+\cos \left(\mathbf{k} \cdot \mathbf{a}_{2}\right) .
$$

These energy dispersion relations correspond to a two dimensional density of states function with typical logarithmic singularities. For finite $N_{3}$, one can calculate numerically $E_{s}$, and therefore $E^{s}(\mathbf{k})$.

\section{NUMERICAL RESULTS}

The expression derived in Sec. III are simple, and manageable to numerical calculations. A graphical solution of Eq. (33), applied to a finite $N_{3}$, is displayed in Fig. 2; the intersection of the horizontal lines, corresponding to various $D$ values, with the $g_{1}(E)+g_{2}(E)$ curves, yields the roots of Eq. (33). However, the numerical solutions of Eq. (33) and (34) are determined by Newton's method. The dependence of the energy levels of the localized states on the environmental shift term $D$, is displayed in Fig. 3. The center curve of Fig. 3 denotes a solution for an infinitely thick crystal [see Eq. (38)], corresponding to two degenerate states. When $N_{3}$ is finite, the two localized states corresponding to Eq. (33) and (34), are nearly degenerate. The curves above the center curve, and below the center curve, correspond to the symmetric, and antisymmetric localized states wavefunctions, respectively. Either by increasing $N_{3}$ or $|D|$, the solutions for the finite crystal approach rapidly the solutions of the infinite crystal. Hence, surface states of thin crystals can be approximated in most cases by considering the solutions for which $N_{3} \rightarrow \infty$. The optical absorption, corresponding to the localized states of thin crystals, can be easily evaluated by inspecting the results given for the infinite crystal, as demonstrated in Fig. 4.

The extent of surface localization is illustrated in Fig. 5. The exciton is almost entirely localized on the two surfaces of the crystal for a large environmental shift

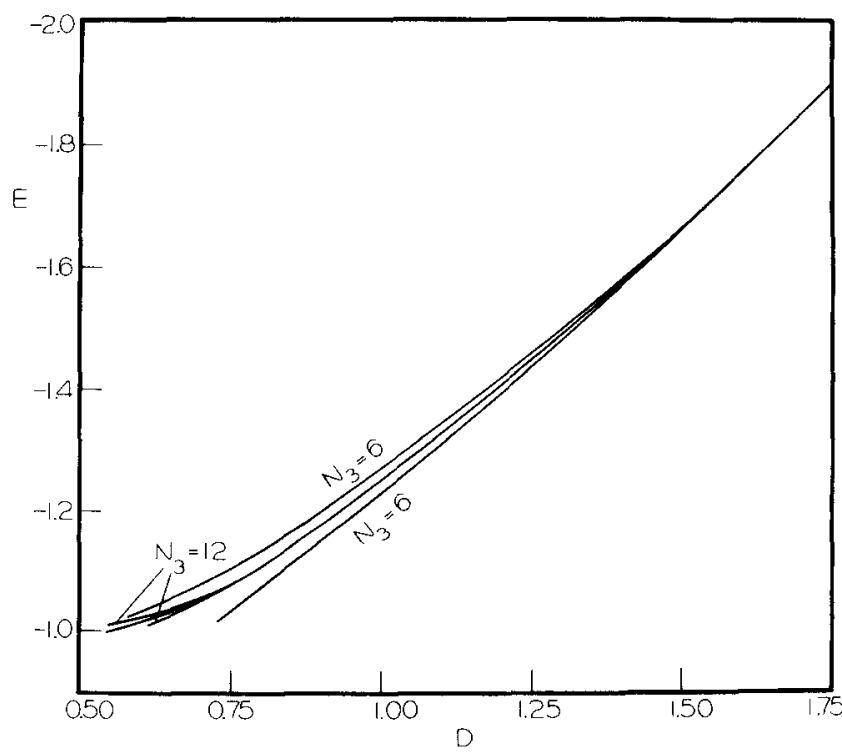

FIG. 3. Energy levels of localized states versus the environmental shift term. The center curve denotes an infinitely thick crystal for which $N_{3} \rightarrow \infty$. Curves above, and below the center curve correspond to solutions of Eq. (33), and (34), respectively. 


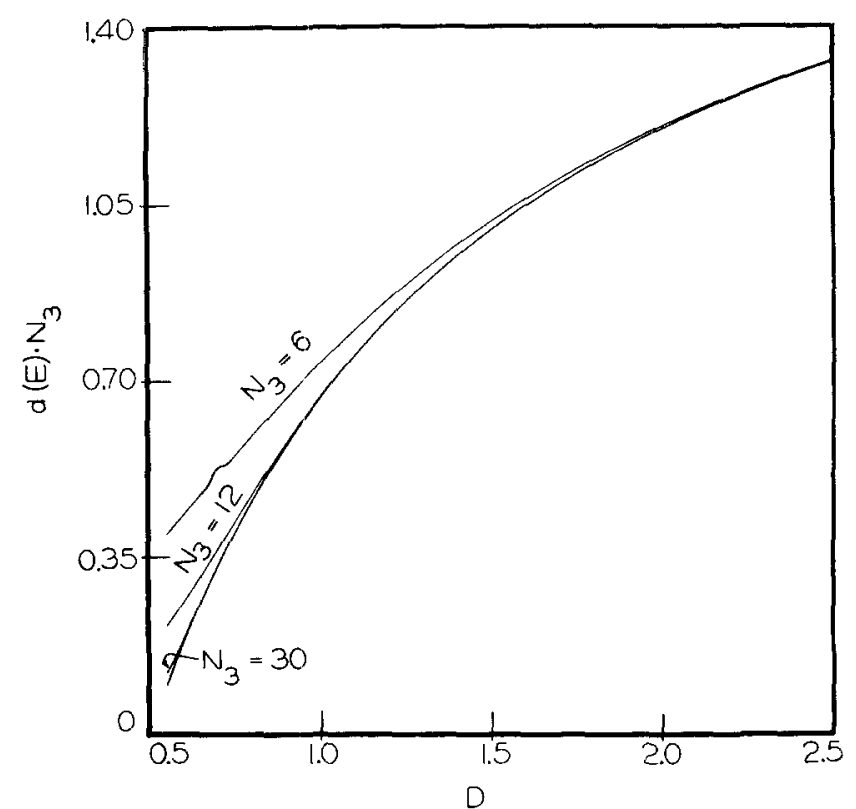

FIG. 4. Dipole strength per unit of energy per molecule $d(E)$, weighed by $N_{3}$. Bottom line denotes the limiting case for which $N_{3} \rightarrow \infty$.

term, whereas partial localization is determined for intermediate $D$ terms for planes adjacent to the surface planes. This partial localization decreases rapidly as $D$ is increased.

The bulk optical absorption is displayed in Fig. 6 for $N=150$, and some values of $D$. These results are compared to the absorption line shapes, given in Fig. 7, of an infinite crystal. The comparison between the thin crystal and the infinitely thick crystal is not as direct as was demonstrated for the localized states. Bulk states of infinitely thick crystals are extended along the energy axis, while bulk states of thin crystals are discrete. Even so, in either case a sharp optical peak is formed at the top of the exciton band.

\section{DISCUSSION}

The pertinent results of this work are related to the possible generation of surface exciton states within the framework of the Frenkel-Davydov ${ }^{6,7}$ theory on molecular excitons. The formation of surface exciton states in molecular crystals depends on the magnitude of the environmental shift terms, relative to the exciton transfer terms. These surface excitons are comparable to electron Tamm states, ${ }^{1}$ in that surface excitons can be predicted for a single exciton band, and a simple crystal structure. The apparent difference between these surface excitons and Tamm's surface electron states is that some kind of surface deformation is assumed for the Tamm electron states. This deformation results in a change of the surface potential parameters relative to the corresponding bulk parameters. The reason for this difference in the results for surface excitons in relation to the results for Tamm's electron states is associated with the multielectron wavefunctions utilized for the molecular exciton, whereas only one-electron crystal wavefunctions are involved in the formation of Tamm's states. Although the calculations presented herein are based on a simple model, some general conclusions may apply to more complex systems. These conclusions are:

(a) Surface exciton states may exist if the environmental shift terms are of the order of magnitude or larger than the exciton transfer terms.

(b) The degree of localization of surface states increases with the increase of the environmental shift terms.

(c) Surface states are not very sensitive to crystal thickness. This situation is more pronounced, when comparatively large environmental shift terms are encountered.

(d) The bulk optical absorption of thick crystals is not effected by the surface, however thin crystals may show some dependence on the thickness of the crystal. On the other hand, a very interesting situation may arise, when the surface states lie below the bulk states. In this case, it may be possible to trap bulk excitons by the surface. This process may be followed by surface emission. While in most cases the surface states will be above the bulk states $(D<0)$, in accordance with the gas-to-crystal red shift, the opposite should be true for blue shifted cases (with short range exciton interactions). Some triplet states in organic crystals are likely candidates. ${ }^{14}$ Actually evidence has been offered by Brodin et al. ${ }^{15}$ for exciton surface states lying above the lowest singlet exciton bulk state in anthracene. This evidence is based on a combination of reflection and fluorescence low temperature spectra. Obviously, slow thermal equilibration, relative to the exciton lifetime, has to be involved. We point out that Brodin et al. ${ }^{15}$ base their interpretation on Sugakov's approach, ${ }^{18}$ as they believe this anthracene exciton band to involve primarily long-range (transition dipole-transition dipole)

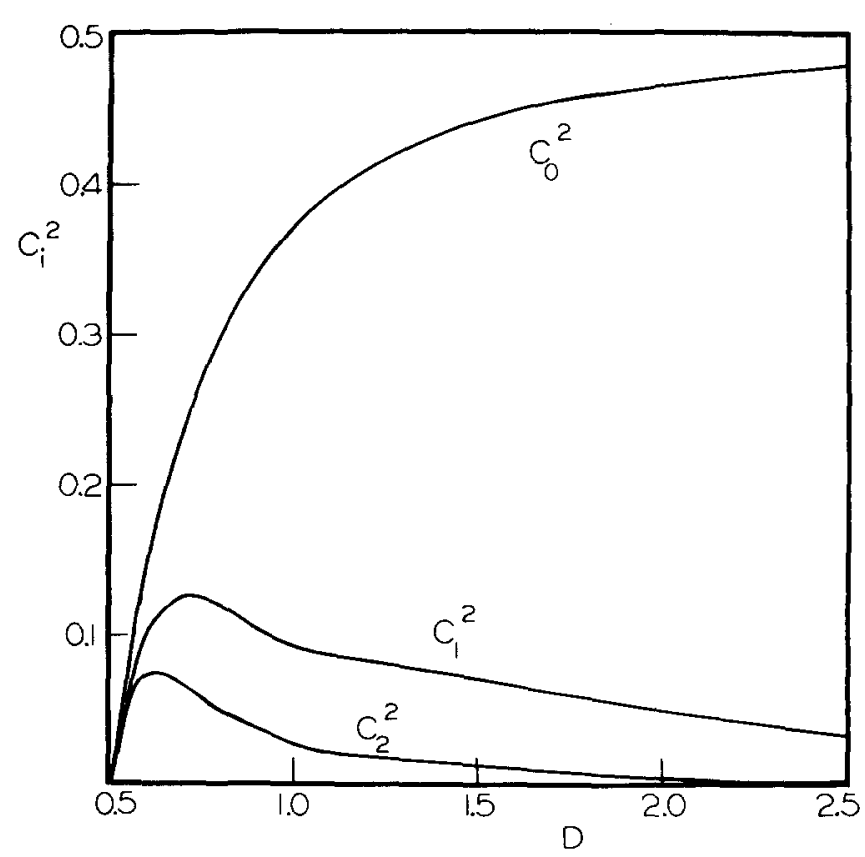

FIG. 5. Localization of excitons on various surface planes. Note that there are no localized states for $|D|<\frac{1}{2}$. 


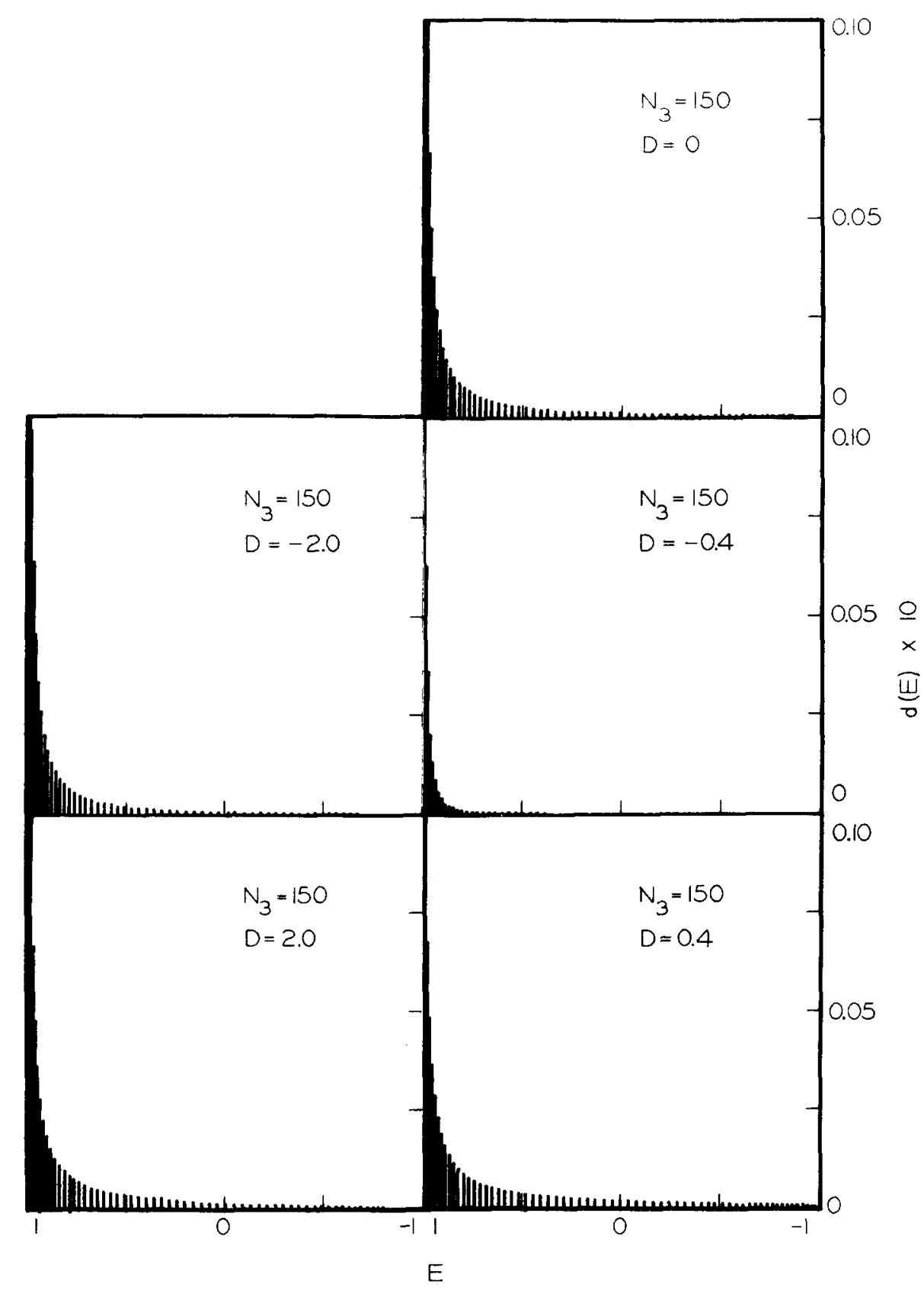

FIG. 6. The dipole strength per unit of energy per molecule for the bulk states, for various values of $D$.

interactions. In spite of formal similarities we note that our theory is more general but our emphasis in applications has been on short-range dynamic (exciton) interactions, as well as short-range static (site-shift) interactions.

To end this discussion of surface excitons, we would like to offer some suggestions which we feel deserve further investigation. Although some of the following suggestions may seem speculative in nature, nevertheless, we believe they pose interesting possibilities. They are:

(a) A theoretical investigation of the effect of exciton surface states on the reflectivity of molecular crystals based on the model presented in this paper, may help to elucidate experimental results related to this model.

(b) A comprehensive study of more complex models may furnish results on the availability of all values of the wavevector $\mathbf{k}$ in two dimensions for a set of surface states. This may be pertinent to the study of optical and transport properties of surface excitons.

(c) Since surface molecules comprise a small fraction of the entire crystal, it may be possible to pump surface excitons in a large concentration by exciting the molecules in the bulk of the crystal, followed by surface trapping. Such a process would be of interest to the study of exciton-exciton interaction.

Finally, it should not be forgotten that our entire discussion involved idealized, flat, surfaces. Real surfaces are extremely varied and sample dependent. What kind of real surfaces would be amenable to our formalism will have to be discovered experimentally.

Note added in proof: Two recently published papers on Green's function theory of surface electron states have just come to our attention [E. N. Foo and H. S. Wong, Phys. Rev. B 9, 1857 (1974); D. Kalkstein and 


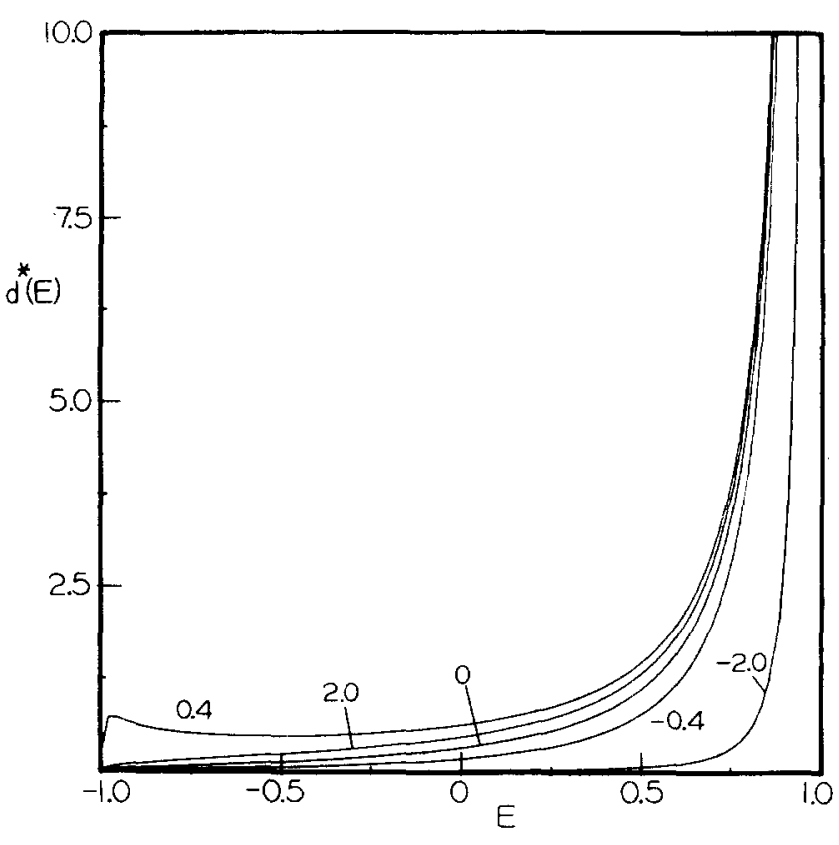

FIG. 7. Line shapes of dipole strength per unit of energy, for various values of $D$, where $d^{*}(E)=N_{3} d(E)$, and $N_{3} \rightarrow \infty$.

P. Soven, Surface Science 26, 86 (1971)]. There are obvious similarities in the Green's function formalism between these works and ours, but we would point out that our more general formalism is for many electron functions, admits long range interactions, and does not depend on an ad hoc surface potential perturbation. In addition, we discuss optical properties. On the other hand, our treatment is addressed specifically to Frenkel excitons, including vibrational and vibronic ones.

\section{APPENDIX}

The transformation of Eq. (52) into Eq. (53) can be accomplished by applying the projection operators

$$
\hat{1}=\sum_{\mathbf{K}}|\mathbf{K}\rangle\langle\mathbf{K}|
$$

on Eq. (A1):

$$
T(E) \equiv\left\langle\psi_{0}|\hat{\mu} \hat{G} \hat{\mu}| \psi_{0}\right\rangle,
$$

where $|\mathbf{K}\rangle$ are the eigenfunction of the unperturbed system belonging to a three dimensional wavevector $K$. Thus

$$
\begin{aligned}
T(E) & =\sum_{\mathbf{K}} \sum_{\mathbf{K}^{\prime}}\left\langle\psi_{0}|\hat{\mu}| \mathbf{K}\right\rangle\left\langle\mathbf{K}|\hat{G}(E)| \mathbf{K}^{\prime}\right\rangle\left\langle\mathbf{K}^{\prime}|\hat{\mu}| \psi_{0}\right\rangle \\
& =\left\langle\psi_{0}|\hat{\mu}| \mathbf{O}\right\rangle\langle\mathbf{O}|\hat{G}(E)| \mathbf{O}\rangle\left\langle\mathbf{O}|\hat{\mu}| \psi_{0}\right\rangle \\
& =N \mu_{0}^{2}\langle\mathbf{O}|\hat{G}(E)| \mathbf{O}\rangle .
\end{aligned}
$$

Utilizing Eq. (18) we obtain for $T(E)$

$$
T(E)=N \mu_{0}^{2}[E-E(O)]^{-1}+N \mu_{0}^{2} S(E),
$$

where $S(E)$ is represented in the form

$$
S(E)=\left\langle\mathrm{O}\left|\hat{G}_{0}(E) \hat{V}\left[\hat{1}-\hat{G}_{0}(E) \hat{V}\right]^{-1} \hat{G}_{0}(E)\right| \mathbf{O}\right\rangle .
$$

$S(E)$ can be further reduced by utilizing the projection operators

$$
\hat{1}=\sum_{\mathbf{k}, l}|\mathbf{k}, l\rangle\langle\mathbf{k}, l|
$$

where $|\mathbf{k}, l\rangle$ is given by $E q$. (1) and

$$
\begin{aligned}
\hat{\mathbf{1}} & =\sum_{\mathbf{k}}|\mathbf{K}\rangle\langle\mathrm{K}\rangle: \\
S(E) & =\sum_{\mathbf{K}} \sum_{\mathbf{K}^{\prime}} \sum_{\mathbf{k}} \sum_{l}\left\langle\mathrm{O}\left|\hat{G}_{0}(E)\right| \mathbf{K}\right\rangle\langle\mathbf{K}|\hat{V}| \mathbf{k}, l\rangle \\
& \otimes\left\langle\mathbf{k}, l\left|\left[\hat{\imath}-\hat{G}_{0}(E) \hat{V}\right]^{-1}\right| \mathbf{K}^{\prime}\right\rangle\left\langle\mathbf{K}^{\prime}\left|\hat{G}_{0}(E)\right| \mathbf{K}\right\rangle \\
& =[E-E(\mathrm{O})]^{2} \sum_{l}\langle\mathbf{O}|\hat{V}| \mathbf{k}=0, l\rangle\left\langle\mathbf{k}=0, l\left|\left[\hat{\mathfrak{l}}-\hat{G}_{0}(E) \hat{V}\right]^{-1}\right| \mathbf{O}\right\rangle .
\end{aligned}
$$

Note that $\hat{G}_{0}(E)$ is diagonal in $|K\rangle$.

Expanding $\langle\mathrm{K}=\mathbf{O}\rangle$ in terms of $|\mathbf{k}=0, l\rangle$ :

$$
|\mathbf{O}\rangle=N_{3}^{-1 / 2} \sum_{l}|0, l\rangle
$$

and inserting in (A5) we obtain

$$
\begin{aligned}
S(E)= & N_{3}^{-1}[E-E(O)]^{-2} \sum_{n, l, m}\langle 0, n|\hat{V}| 0, l\rangle \\
& \otimes\left\langle 0, l\left|\left(1-\hat{G}_{0}(E) \hat{V}\right)^{-1}\right| 0, m\right\rangle .
\end{aligned}
$$

By dropping zero terms form (A7) the following expression is derived for $S(E)$ :

$$
\begin{aligned}
S(E)= & N_{3}^{-1}[E-E(\mathrm{O})]^{-2}\left(V_{11} R_{00}+V_{00} R_{01}+V_{01} R_{10}+V_{01} R_{11}\right. \\
& \left.+V_{10} R_{00}+V_{10} R_{01}+V_{11} R_{10}+V_{11} R_{11}\right)
\end{aligned}
$$

where

$$
V_{i j}=\langle 0, i|\hat{V}| 0, j\rangle
$$

and

$$
R_{i j}=\langle 0, i|\hat{R}| 0, j\rangle \text { and } \hat{R}
$$

is given as

$$
\hat{R}=\left[1-\hat{G}_{0}(E) \hat{V}\right]^{-1}
$$

Note that $\hat{V}$ connects only adjacent surface planes. The matrix elements $R_{i j}$ and $V_{i j}$ of Eq. (A8) are given in the form:

$$
\begin{aligned}
R_{00}= & R_{11}=\left[1+g_{0}(E) D+g_{1}(E) J\right]\left\{\left[1+\left(g_{0}(E)+g_{1}(E)\right)(D+J)\right]\right. \\
& \left.\otimes\left[1+\left(g_{0}(E)-g_{1}(E)\right)(D-J)\right]\right\}^{-1}, \\
R_{01}= & R_{10}=-\left[g_{0}(E) J+g_{1}(E) D\right]\left\{\left[1+\left(g_{0}+g_{1}\right)(D+J)\right]\right. \\
& \left.\otimes\left[1+\left(g_{0}-g_{1}\right)(D-J)\right]\right\}^{-1} \\
& V_{00}=V_{11}=D \\
V_{01} & =V_{10}=J
\end{aligned}
$$

Plugging Eqs. (A9), (A10), (A11), and (A12) into Eq. (A8) the following expression is derived for $S(E)$ :

$$
S(E)=\frac{-2(D+J)}{N_{3}[E-E(\mathbf{O})]^{2}\left\{1+\left[g_{0}(E)+g_{1}(E)\right](D+J)\right\}} .
$$


*Supported by NSF Grant GP-18718 and NIH Grant NS08116.

${ }^{1}$ I. Tamm, Phys. Z. Sowjetunion 1, 733 (1932).

${ }^{2}$ W. Shockley, Phys. Rev. 56, 317 (1939).

${ }^{3}$ J. C. Koutecky, Angew. Chem. Int. Ed. Engl. 3, 496 (1964);

Phys. Status Solidi 1, 554 (1961); Phys. Rev. 108, 13 (1957).

${ }^{4} \mathrm{~T}$. B. Grimley, Adv. Catal. 12, 1 (1960).

${ }^{5}$ P. S. Stern and M. E. Green, J. Chem. Phys. 58, 2507 (1973).

6. Frenkel, Phys. Rev. 37, 17 (1931).

${ }^{7}$ A. S. Davydov, Zh. Eksper. Teor. Fiz. 18, 210 (1948).

${ }^{8} \mathrm{~J}$. Hoshen and J. Jortner (to be published); J. Hoshen, Ph. D. thesis, Tel-Aviv University (1971).

${ }^{9}$ H. C. Bolton, W. Fawcett, and D. C. Gurney, Proc. Phys. Soc. Lond. A 80, 199 (1962).

${ }^{10}$ F. W. de Wette and G. E. Schacher, Phys. Rev. 137, A78,
A92 (1965).

${ }^{11}$ M. R. Philpott, J. Chem. Phys. 58, 588 (1973).

${ }^{12}$ G. F. Koster and J. C. Slater, Phys. Rev. 95, 1167 (1954); 96,1208 (1954).

${ }^{13}$ B. Sommer and J. Jortner, J. Chem. Phys. 50, 187 (1969).

${ }^{11}$ For instance, while the first excited singlet state of benzene is red shifted by $248 \mathrm{~cm}^{-1}$ [E. R. Bernstein, S. D. Colson, R. Kopelman, and G. W. Robinson, J. Chem. Phys. 48, 5596 (1968)], the first excited triplet is blue shifted by about 110 $\mathrm{cm}^{-1}$ [D. M. Burland, G. Castro, and G. W. Robinson, J. Chem. Phys, 52, 4100 (1970)].

${ }^{15}$ M. S. Brodin, M. A. Dudinskii, and S. V. Marisova, Opt. Spectrose. 31, 401 (1971).

${ }^{16}$ V. I. Sugakov, Ukr. Fiz. Zh. 14, 1425 (1969); 15, $2060(1970)$. 